\title{
Stochastic Modeling for HIV and AIDS Epidemics With Viral Load Detectability
}

\author{
Peter Emeda Tengaa ${ }^{1}$, Samuel Mwalili ${ }^{2}$, George Otieno Orwa ${ }^{3}$ \\ ${ }^{1}$ Department of Mathematics and Statistics, Pan African University Institute of Basic Sciences Technology and Innova- \\ tion, Nairobi, Kenya \\ ${ }^{2}$ Department of Statistics and Actuarial Sciences, Jomo Kenyatta University of Agriculture and Technology, Nairobi, \\ Kenya \\ ${ }^{3}$ Department of Mathematics and Statistics, Bomet University College,Bomet, Kenya \\ Correspondence: Peter Emeda Tengaa, Department of Mathematics and Statistics, Pan African University Institute of \\ Basic Sciences Technology and Innovation, P. O. Box 62000 00200, Nairobi, Kenya. E-mail: emedason@gmail.com
}

Received: April 6, 2020 Accepted: May 28, 2020 Online Published: June 9, 2020

doi:10.5539/ijsp.v9n4p33

URL: https://doi.org/10.5539/ijsp.v9n4p33

\begin{abstract}
In this paper, we investigated on the stochastic epidemic model by incorporating viral load detectability. We derived HIV and AIDS stochastic model from the deterministic counterpart model and presented a stochastic threshold in terms of stochastic basic reproduction number. We showed that $R_{0}^{s}<1$ then the disease dies out exponentially and when $R_{0}^{s}>1$ the disease persists in the population. We further derived the existence and uniqueness, extinction and persistence properties of the stochastic model models, then the numerical simulation is done by using Milsten's numerical scheme. The finding shows that the random perturbation introduced in the stochastic model suppresses disease outbreak as compared to its deterministic counterparts which provide some useful control strategies to eradicate the disease.
\end{abstract}

Keywords: stochastic epidemic model, existence, extinction, persistence, viral load detectability and itô formula

\section{Introduction}

As in the early stages of an epidemic, the number of infectious will be very small, randomness alone can be the source of an epidemic to perish out, and it is much more significant particularly to include perturbation into models. Stochastic models possibly will be an additional suitable technique for modelling disease epidemics in many circumstances which gives a better understanding of transmission dynamics derived from its deterministic counterpart. It has similarly been revealed that some stochastic epidemic models can deliver an extra degree of realism in contrast to the deterministic model. Explicitly, the stochastic model suits well for disease extinction and disease persistence (Van Herwaarden and Grasman, 1995).

There is a fair quite a number literature about stochastic models, for example, authors such as Cai et al. (2016) and Mao et al. (2002) studied deterministic and stochastic models for ratio dependents transmission rates with the main focus on how environmental perturbations particularly affects the dynamics of the diseases and others focus on the environment with media effect. Also, Zhang et al. (2014), developed and gave an excellent clear investigation of non-linear incidence rate, and analyzed the extinction, persistence, and stationary distribution of stochastic disease epidemic model. Similarly, Song et al. (2018) developed SIRS stochastic model and particularly investigated extinction and persistence with saturated incidence rate, most importantly they showed that large noise in the model reduces disease outbreak. Stochastic models could be a more suitable way of modelling epidemics in many situations and many realistic stochastic models can be deriving from their deterministic counterparts (Fan et al., 2017; Ji and Jiang, 2017; Zhang and Zhou, 2019; Zhao and Yuan, 2016; Witbooi, 2013).

There are numerous ways of deriving a stochastic model from the deterministic model: parametric perturbation stochastic model which introduces diffusion coefficient of the specific parameter(s) of interest to be perturbed, while on other hands non-parametric perturbation stochastic model involves adding Brownian processes to each differentia equation and assume that each compartment has uncertainty (Nsengiyumva et al., 2013; N'zi and Kanga, 2016; Miao et al., 2017). Several authors have undertaken various stochastic models for HIV and AIDS including authors such as (Mbogo et al., 2013; Zhang and Zhou, 2019; Fan et al., 2017). However, none of these models have considered stochastic models for HIV and AIDS particular by incorporating viral load detectability in the model, and the stochastic model is derived from deterministic counterpart as considered by Tengaa et al. (2020). This paper is arranged as follows:- In section 2, we 
formulated a stochastic model from deterministic as considered by Tengaa et al. (2020). In section 3, we investigate the quantitative analysis of the model such as deriving the existence and uniqueness, extinction, and persistence properties of the stochastic model models. Section 4 shows the numerical solution of the model and lastly, in section 5 is the concluding remark.

\section{Model Formulation}

\subsection{Description of the Model}

The model is divided into five compartmental models which includes Susceptibles population $\left(S_{t}\right)$, Infected population $\left(I_{t}\right)$, AIDS population on treatment $\left(A_{t}\right)$, Detectable $\left(D_{t}\right)$ and Undetectable $\left(U_{t}\right)$ viral load population. The frequency dependent transmission is assumed with force of infection $\lambda=\beta \frac{I}{N(t)}$, where $\beta$ is the rate of transmission. $I_{t}$ can be increase by the proportion $(1-\rho)$ from infected mothers who give birth to infected children, also $I_{t}$ can progress to $A_{t}$ with a rate of $\theta_{1}$. Moreover, $A_{t}$ after failure of therapy they can either join $D_{t}$ with detectable viral load failure rate of $\psi$ or can join $U_{t}$ with a rate of $(1-\psi)$. In turn with HIV and AIDS viral load test or drug resistance tests while also HIV replicates at an extremely rapid rate then individuals in $D_{t}$ can be treated with a rate $\theta_{2}$ and individuals in $U_{t}$ can be treated with a rate of $\theta_{3}$ to move back to $A_{t}$ on treatment class, whilst $\mu$ is the natural mortality rate while $\alpha$ is the death due to AIDS individuals. The normalized deterministic model as considered by Tengaa et al. (2020)

$$
\left\{\begin{array}{l}
\frac{\mathrm{d} s}{\mathrm{~d} t}=\rho \Lambda-[\beta i+\Lambda-\alpha(a+d+u)] s \\
\frac{\mathrm{d} i}{\mathrm{~d} t}=(1-\rho) \Lambda+\left[\beta s-\theta_{1}-\Lambda+\alpha(a+d+u)\right] i \\
\frac{\mathrm{d} a}{\mathrm{~d} t}=\theta_{1} i+\theta_{2} d+\theta_{3} u-[\alpha+\omega+\Lambda-\alpha(a+d+u)] a \\
\frac{\mathrm{d} d}{\mathrm{~d} t}=\psi \omega a-\left[\alpha+\theta_{2}+\Lambda-\alpha(a+d+u)\right] d \\
\frac{\mathrm{d} u}{\mathrm{~d} t}=(1-\psi) \omega a-\left[\alpha+\theta_{3}+\Lambda-\alpha(a+d+u)\right] u
\end{array}\right.
$$

subject to condition $s+i+a+d+u=1$. All the feasible solutions of system (2.1) enters the region of biological interest defined by

$$
\Omega=\left\{(s, i, a, d, u) \in \mathfrak{R}_{+}^{5}: s+i+a+d+u=1\right\}
$$

that is positive-invariant solution. We consider the dynamics of the flow generated by system (2.1) in $\Omega$. In this region, therefore the model (2.1) is considered to be both biologically and mathematically well posed with a deterministic reproduction number given as $R_{0}^{d}=\frac{\beta \rho}{\left(\theta_{1}+\Lambda\right)}$ as used by Tengaa et al. (2020).

We introduce randomness into model system (2.1) by replacing the parameters $\beta$ and $\omega$ by $\beta \longrightarrow \beta+\sigma_{1} d B_{1}$ and $\omega \longrightarrow$ $\omega+\sigma_{2} d B_{2}$ respectively which is parametric perturbation stochastic model.

Therefore, the stochastic model for nondimensionalized system of equations (2.1) becomes:

$$
\left\{\begin{array}{l}
d s=[\rho \Lambda-(\beta i+\Lambda-\alpha(a+d+u)) s] d t-\sigma_{1} i s d B_{1}(t) \\
d i=\left[(1-\rho) \Lambda+\left(\beta s-\theta_{1}-\Lambda+\alpha(a+d+u)\right) i\right] d t+\sigma_{1} i s d B_{1}(t) \\
d a=\left[\theta_{1} i+\theta_{2} d+\theta_{3} u-(\alpha+\omega+\Lambda-\alpha(a+d+u)) a\right] d t-\sigma_{2} a d B_{2}(t) \\
d \mathrm{~d}=\left[\psi \omega a-\left(\alpha+\theta_{2}+\Lambda-\alpha(a+d+u)\right) d\right] d t+\sigma_{2} \psi a d B_{2}(t) \\
d u=\left[(1-\psi) \omega a-\left(\alpha+\theta_{3}+\Lambda-\alpha(a+d+u)\right) u\right] d t+\sigma_{2}(1-\psi) a d B_{2}(t)
\end{array}\right.
$$

where $B_{i}(t)(i=1,2)$ are independent standard Brownian Motions and $\sigma_{i}(i=1,2)$ are constant intensities of environmental fluctuations respectively. We define bounded set $\Omega$ as

$$
\Omega=\left\{(s, i, a, d, u) \in \mathfrak{R}_{+}^{5}: 0<(s+i+a+d+u) \leq 1\right\}
$$

Throughout this paper, let $(\Omega, \mathcal{F}, P)$ be a complete probability space with filtration $\left\{\mathcal{F}_{t}\right\}_{t \in \mathfrak{R}_{+}}$satisfying the usual conditions(right continous and increasing while $\mathcal{F}_{0}$ contains all P-null sets). We denote as

$$
\mathbf{x}(t)=(s, i, a, d, u):=\left(x_{1}(t), x_{2}(t), x_{3}(t), x_{4}(t), x_{5}(t)\right)
$$

and denote $C^{2,1}\left(\mathfrak{R}^{5} \times(0, \infty): \mathfrak{R}_{+}\right)$as the family of all nonnegative functions $V(\mathbf{x}, t)$ defined on $\mathfrak{R}^{5} \times(0, \infty)$ such that they are continously twice differentiable in $\mathbf{x}$ and differentiable once in $t$. 


\section{Model Analysis}

\subsection{Existence and Uniqueness of Stochastic Model}

To investigate the behaviour of the system (2.2) we need to consider the system is positive and ensure the existence of global behaviour. In order to get stochastic model which has unique global solution(no explosion in finite time) for a given initial values, the coefficient of the equation are required to satisfy the linear growth condition and the Lipschitz condition. But according to system (2.2), the linear growth condition is not satisfied, but the system is locally Lipschitz and the solution of the system (2.2) may explode at finite time.

Theorem 3.1. There is a unique solution $(s(t), i(t), a(t), d(t), u(t))$ of system (2.2) $t \geq 0$ for any initial value

$$
(s(0), i(0), a(0), d(0), u(0)) \in \mathfrak{R}_{+}^{5}
$$

and the solution remain in $\mathfrak{R}_{+}^{5}$ with probability 1 , namely, $(s(t), i(t), a(t), d(t), u(t)) \in \mathfrak{R}_{+}^{5}$ for all $t \geq 0 \quad$ a.s.

Proof. Since the coefficients of the equation are locally Lipschitz continuous for any given initial value

$$
(s(0), i(0), a(0), d(0), u(0)) \in \mathfrak{R}_{+}^{5}
$$

, there is a unique local solution $(s(t), i(t), a(t), d(t), u(t))$ on $t \in\left(0, \tau_{e}\right)$ where $\tau_{e}$ is the explosion time. To show that the solution of the system (2.2) is global, we need to show that $\tau_{e}=\infty$ a.s. Let $k_{0} \geq 0$ be sufficiently large such that $(s(0), i(0), a(0), d(0), u(0))$ lies within the interval $\left[\frac{1}{k_{0}}, k_{0}\right]$. For each integer $k \geq k_{0}$ we define the stopping time as follows

$$
\begin{gathered}
\tau_{k}=\inf \left\{t \in\left[0, \tau_{e}\right]: \min \{(s(t), i(t), a(t), d(t), u(t))\} \leq \frac{1}{k} \quad\right\} \\
\text { or } \max \{(s(t), i(t), a(t), d(t), u(t))\} \geq k
\end{gathered}
$$

Note that according to definition, $\tau_{k}$ is increasing as $k \longrightarrow \infty$, by setting $\tau_{\infty}=\lim _{k \rightarrow \infty} \tau_{k}$, where $\tau_{\infty} \leq \tau_{e}$ a.s. Required to show $\tau_{\infty}=\infty$ a.s, then $\tau_{e}=\infty$ and $(s(t), i(t), a(t), d(t), u(t)) \in \mathfrak{R}_{+}^{5}$ a.s. But if this condition is false, then there exist a pair of constants $T>0$ and $\epsilon \in(0,1)$ such that

$$
P\left\{\tau_{\infty} \leq T\right\}>\epsilon
$$

Therefore, there is an integer $k_{1} \geq k_{0}$ such that

$$
\begin{gathered}
P\left\{\tau_{k} \leq T\right\} \geq \epsilon, \text { for } t \leq \tau_{k}, \text { for each } k \\
d(s(t), i(t), a(t), d(t), u(t))=[\rho \Lambda-(\beta i+\Lambda-\alpha(a+d+u)) s] d t-\sigma_{1} i s d B_{1}(t) \\
+\left[(1-\rho) \Lambda+\left(\beta s-\theta_{1}-\Lambda+\alpha(a+d+u)\right) i\right] d t+\sigma_{1} i s d B_{1}(t) \\
+\left[\theta_{1} i+\theta_{2} d+\theta_{3} u-(\alpha+\omega+\Lambda-\alpha(a+d+u)) a\right] d t-\sigma_{2} a d B_{2}(t) \\
+\left[\psi\left(\omega a-\left(\alpha+\theta_{2}+\Lambda-\alpha(a+d+u)\right) d\right] d t+\sigma_{2} \psi a d B_{2}(t)\right. \\
+\left[(1-\psi) \omega a-\left(\alpha+\theta_{3}+\Lambda-\alpha(a+d+u)\right) u\right] d t+\sigma_{2}(1-\psi) a d B_{2}(t)
\end{gathered}
$$

Upon simplifying we get;

$$
\begin{aligned}
d(s(t), i(t), a(t), d(t), u(t)) & =[\Lambda-\Lambda(a+d+i+s+u)+ \\
& \alpha(a+d+u)(1-(a+d+i+s+u))] d t
\end{aligned}
$$

and then,

$$
d(s(t), i(t), a(t), d(t), u(t)) \leq[\Lambda+\alpha(a+d+u)(1-(a+d+i+s+u))] d t
$$

Let $Z=(s(t), i(t), a(t), d(t), u(t))$ then

$$
Z=\left\{\begin{array}{clc}
\frac{\Lambda}{\alpha} & \text { if } & s(0)+i(0)+a(0)+d(0)+u(0) \leq \frac{\Lambda}{\alpha} \\
s(0)+i(0)+a(0)+d(0)+u(0) & \text { if } & s(0)+i(0)+a(0)+d(0)+u(0)>\frac{\Lambda}{\alpha}
\end{array}\right.
$$

Let $C^{2,1}$ be twice continuously differentiable in $\mathbf{x}$ and once continuously differentiable in $t$ and let lyapunov function candidate be $V: \mathfrak{R}_{+}^{5} \longrightarrow \mathfrak{R}_{+}$by

$$
V(s, i, a, d, u)=(s-1-\log s)+(i-1-\log i)+(a-1-\log a)+(d-1-\log d)+(u-1-\log u)
$$


We define the differential oparetor $\mathbf{L}$ associated with 5-dimensional stochastic differential equations $d \mathbf{x}=f(\mathbf{x}, t) d t+$ $g(\mathbf{x}, t) d B(t)$ as

$$
\mathbf{L}=\frac{\partial}{\partial t}+\sum_{i=1}^{5} f_{i}(x, t) \frac{\partial}{\partial x_{i}}+\frac{1}{2} \sum_{i, j=1}^{5}\left[g^{T} g(x, t)\right]_{i, j} \frac{\partial^{2}}{\partial x_{i} \partial x_{j}}
$$

If $\mathbf{L}$ acts on a function $V \in C^{2,1}\left(\mathfrak{R}^{5} \times(0, \infty): \mathfrak{R}_{+}\right.$then we denote

$$
L V(x, t)=V_{t}(x, t)+V_{x}(x, t) f(x, t)+\frac{1}{2} \operatorname{trace}\left[g^{T}(\mathbf{x}, t) V_{x x}(\mathbf{x}, t)\right]
$$

where $T$ means transposition, $V_{t}=\frac{\partial V}{\partial t}, V_{x}=\left(\frac{\partial V}{\partial x_{1} \cdots}, \frac{\partial V}{\partial x_{5}}\right), V_{x x}=\left(\frac{\partial^{2} V}{\partial x_{i} \partial x_{j}}\right)_{5 \times 5}$. By Itô general formula we have

$$
\begin{aligned}
& d V(s, i, a, d, u)=\frac{\partial V(s, i, a, d, u)}{\partial t} d t+\sum_{i=1}^{5} \frac{\partial V(s, i, a, d, u)}{\partial X i} d X i+\frac{1}{2} \sum_{i=1}^{5} \frac{\partial^{2} V(s, i, a, d, u)}{\partial X i} d[X i]_{t} \\
& d V(s, i, a, d, u)=\mathcal{L} V_{1} d t+\sigma_{i} \mathrm{~dB}(t) \\
& \sum_{i=1}^{5} \frac{\partial V(s, i, a, d, u)}{\partial X i} d X i=\frac{\partial V}{\partial s} d s_{t}+\frac{\partial V}{\partial i} d i_{t}+\frac{\partial V}{\partial a} d a_{t}+\frac{\partial V}{\partial d} d d_{t}+\frac{\partial V}{\partial u} d u_{t} \\
& \quad=\left(1-\frac{1}{s}\right)[\rho \Lambda-(\beta i+\Lambda-\alpha(a+d+u)) s] d t-\left(1-\frac{1}{s}\right) \sigma_{1} i s d B_{1}(t) \\
& \quad+\left(1-\frac{1}{i}\right)\left[(1-\rho) \Lambda+\left(\beta s-\theta_{1}-\Lambda+\alpha(a+d+u)\right) i\right] d t+\left(1-\frac{1}{i}\right) \sigma_{1} i s d B_{1}(t) \\
& \quad+\left(1-\frac{1}{a}\right)\left[\theta_{1} i+\theta_{2} d+\theta_{3} u-(\alpha+\omega+\Lambda-\alpha(a+d+u)) a\right] d t-\left(1-\frac{1}{a}\right) \sigma_{2} a d B_{2}(t) \\
& \quad+\left(1-\frac{1}{d}\right)\left[\psi \omega a-\left(\alpha+\theta_{2}+\Lambda-\alpha(a+d+u)\right) d\right] d t+\left(1-\frac{1}{d}\right) \sigma_{2} \psi a d B_{2}(t) \\
& \quad+\left(1-\frac{1}{u}\right)\left[(1-\psi) \omega a-\left(\alpha+\theta_{3}+\Lambda-\alpha(a+d+u)\right) u\right] d t+\left(1-\frac{1}{u}\right) \sigma_{2}(1-\psi) a d B_{2}(t)
\end{aligned}
$$

Simplifying gives

$$
\begin{aligned}
\sum_{i=1}^{5} & \frac{\partial V(s, i, a, d, u)}{\partial X i} d X i=\rho \Lambda+\Lambda-\frac{\rho \Lambda}{s}-\beta s i+\beta i-\Lambda s+\alpha s(a+d+u)- \\
& \alpha(a+d+u)+2 \Lambda+\theta_{1}-\frac{(\Lambda-\rho \Lambda)}{i}+\beta s i-\theta_{1} i-\Lambda i+\alpha i(a+d+u)-\beta s \\
& -\alpha s(a+d+u)+\theta_{1} i-\left(\frac{\theta_{1} i+\theta_{2} d+\theta_{3} u}{a}\right)-\alpha a-\omega a-\Lambda a+\alpha a(a+d+u)+\alpha \\
& +\omega+\Lambda-\alpha(a+d+u)-\frac{\psi \omega a}{d}+\frac{\psi \omega a}{u}-\alpha d-\alpha u-\Lambda d-\Lambda u+\alpha d(a+d+u)+ \\
& \alpha u(a+d+u)+2 \alpha+\theta_{3}+\theta_{2}+3 \Lambda-3 \alpha(a+d+u) \\
\sum_{i=1}^{5} & \frac{\partial V(s, i, a, d, u)}{\partial X i} d X i \leq 6 \Lambda+\rho \Lambda+\omega+2 \alpha+\theta_{3}+\theta_{2}+\theta_{1}-(4 \alpha+\omega+5 \Lambda) \mathcal{M}- \\
& (s-1) \sigma_{1} i d B_{1}+(i-1) \sigma_{1} s d B_{1}+(a-1) \sigma_{2} a d B_{2}+(d-1) \sigma_{2} \psi a d B_{2}+ \\
& (u-1) \sigma_{2}(1-\psi) a d B_{2}
\end{aligned}
$$

Similarly the quadratic variation becomes;

$$
\begin{gathered}
\frac{1}{2} \sum_{i=1}^{5} \frac{\partial^{2} V(s, i, a, d, u)}{\partial X i} d[X i]_{t}=-\frac{1}{2}\left[\frac{1}{s^{2}} d[s]_{t}+\frac{1}{i^{2}} d[i]_{t}+\frac{1}{a^{2}} d[a]_{t}+\frac{1}{d^{2}} d[d]_{t}+\frac{1}{u^{2}} d[u]_{t}\right] \\
=-\frac{1}{2}\left[\sigma_{1}^{2} i^{2}+\sigma_{1}^{2} s^{2}+\sigma_{2}^{2}+\frac{\sigma_{2}^{2} \psi^{2} a^{2}}{d^{2}}+\frac{\sigma_{2}^{2}\left(1-\psi^{2}\right) a^{2}}{u^{2}}\right] d t
\end{gathered}
$$


From equation (3.4), substituting (3.5) and (3.6) and considering the drift part we get

$$
\begin{aligned}
& L \mathcal{V} \leq\left[\Lambda+\rho \Lambda+\omega+2 \alpha+\theta_{3}+\theta_{2}+\theta_{1}-(4 \alpha+\omega+5 \Lambda) \mathcal{M}-\frac{1}{2}\left(\sigma_{1}^{2} i^{2}+\sigma_{1}^{2} s^{2}+\sigma_{2}^{2}+\right.\right. \\
& \left.\left.\quad \frac{\sigma_{2}^{2} \psi^{2} a^{2}}{d^{2}}+\frac{\sigma_{2}^{2}\left(1-\psi^{2}\right) a^{2}}{u^{2}}\right)\right] d t \\
& L \mathcal{V} \leq \Lambda+\rho \Lambda+\omega+2 \alpha+\theta_{3}+\theta_{2}+\theta_{1}-(4 \alpha+\omega+5 \Lambda) \mathcal{M}:=\mathbf{K}
\end{aligned}
$$

where, $\mathcal{M}$ is a positive constant and $\mathbf{K}$ is a constant which independent of variables, therefore from (3.4) we have;

$$
\begin{aligned}
& d V=\mathbf{K} d t-(s-1) \sigma_{1} i d B_{1}+(i-1) \sigma_{1} s d B_{1}+(a-1) \sigma_{2} a d B_{2}+(d-1) \sigma_{2} \psi a d B_{2}+ \\
& \quad(u-1) \sigma_{2}(1-\psi) a d B_{2}
\end{aligned}
$$

Integrating both sides of (3.7) from 0 to $\tau_{k} \wedge T$, for $\tau_{k} \wedge T=\min \left\{\tau_{k}, T\right\}$ we have

$$
\begin{aligned}
\int_{0}^{\tau_{k} \wedge T} d V & \int_{0}^{\tau_{k} \wedge T} \mathbf{K} d r-\int_{0}^{\tau_{k} \wedge T}(s-1) \sigma_{1} i d B_{1}(r)+\int_{0}^{\tau_{k} \wedge T}(i-1) \sigma_{1} s d B_{1}(r)+ \\
& \int_{0}^{\tau_{k} \wedge T}(a-1) \sigma_{2} a d B_{2}(r)+\int_{0}^{\tau_{k} \wedge T}(d-1) \sigma_{2} \psi a d B_{2}(r)+ \\
& \int_{0}^{\tau_{k} \wedge T}(u-1) \sigma_{2}(1-\psi) a d B_{2}(r)
\end{aligned}
$$

Applying expectation both sides gives

$$
\begin{aligned}
\mathbb{E}\left[V \left(s\left(\tau_{k} \wedge T\right), i\left(\tau_{k} \wedge T\right),\right.\right. & \left.\left.a\left(\tau_{k} \wedge T\right), d\left(\tau_{k} \wedge T\right), u\left(\tau_{k} \wedge T\right)\right)\right] \leq V(s(0), i(0), a(0) \\
& d(0), u(0))+\mathbf{K} \mathbb{E} \tau_{k} \wedge T \\
& \leq V(s(0), i(0), a(0), d(0), u(0))+\mathbf{K} \mathbb{E} \tau_{k} \wedge T \\
& \leq V(s(0), i(0), a(0), d(0), u(0))+\mathbf{K} T
\end{aligned}
$$

Set $\Omega_{k}=\left\{\tau_{k} \leq T\right\}$ for $k \geq k_{1}$ and by (3.2), $P\left(\Omega_{k}\right) \geq \epsilon$, and note that for every $\omega \in \Omega_{k}$, there is atleast one of $s\left(\tau_{k} \wedge\right.$ $\omega), i\left(\tau_{k} \wedge \omega\right), a\left(\tau_{k} \wedge \omega\right), d\left(\tau_{k} \wedge \omega\right)$ and $u\left(\tau_{k} \wedge \omega\right)$ that equals to either $k$ or $\frac{1}{k}$ and thus $V\left(s\left(\tau_{k} \wedge \omega\right), i\left(\tau_{k} \wedge \omega\right), a\left(\tau_{k} \wedge\right.\right.$ $\left.\omega), d\left(\tau_{k} \wedge \omega\right), u\left(\tau_{k} \wedge \omega\right)\right)$ is not less than

$$
k-1-\log k \text { or } \quad \frac{1}{k}-\log \left(\frac{1}{k}\right)=\frac{1}{k}-1+\log k
$$

Consequently, $V\left(s\left(\tau_{k} \wedge \omega\right), i\left(\tau_{k} \wedge \omega\right), a\left(\tau_{k} \wedge \omega\right), d\left(\tau_{k} \wedge \omega\right), u\left(\tau_{k} \wedge \omega\right) \geq[k-1-\log k] \wedge\left[\frac{1}{k}-\log k\right]\right.$

It then follows from (3.2) and (3.9) that

$$
\begin{aligned}
V(s(0), i(0), a(0), d(0), u(0))+\mathbf{K} T & \geq \mathbb{E}\left[\mathbb { 1 } _ { \Omega _ { k } } ( \omega ) V \left(s\left(\tau_{k} \wedge \omega\right), i\left(\tau_{k} \wedge \omega\right), a\left(\tau_{k} \wedge \omega\right),\right.\right. \\
& \left.\left.d\left(\tau_{k} \wedge \omega\right), u\left(\tau_{k} \wedge \omega\right)\right)\right] \\
& \geq \epsilon\left[(k-1-\log k) \wedge\left(\frac{1}{k}-1+\log k\right)\right]
\end{aligned}
$$

where $\mathbb{1}_{\Omega_{k}}$ is the indicator function of $\Omega_{k}$. By letting $k \longrightarrow \infty$ results to the contradiction $\infty>V(s(0), i(0), a(0), d(0), u(0))+$ $\mathbf{K} T=\infty$. We must therefore have $\tau_{\infty}=\infty$ almost surely.

\subsection{Stochastic Boundedness}

We are required to show that for any initial condition $X(0) \in \mathfrak{R}_{+}^{5}$ the solution of model (2.2) is always positive and remains in $\mathfrak{R}_{+}^{5}$

Definition 3.1. The solution $X(t)$ of the system (2.2) is called stochastically ultimately bounded or ultimately bounded in probability if for any $\epsilon \in(0,1)$ there is a constant $\mathbb{X}=\mathbb{X}(\epsilon>0)$ such that for any initial solution $X(0) \in \Gamma$, then the solution of the system (2.2) has the property that

$$
\limsup _{t \rightarrow \infty} \mathbb{P}\{\|X(t)\|>\mathbb{X}\} \leq \epsilon
$$


Lemma 3.3. For any initial value $X(0) \in \Gamma$ and $\theta>1, \exists \mathbf{k}=\mathbf{k}(\theta)$ such that the solution of the system (2.2) satisfies condition

$$
\limsup _{t \rightarrow \infty} \mathbb{E}\left\{\|X(t)\|^{\theta}\right\}<\mathbf{k}(\theta)
$$

Proof. Let $V\left(s_{t}, i_{t}\right)=e^{t}\left(s^{\theta}+i^{\theta}+a^{\theta}+d^{\theta}+u^{\theta}\right)$, then by using Itô formula, we have

$$
\begin{aligned}
d V & =\frac{\partial V}{\partial t} d t+\frac{\partial V}{\partial X} d X+\frac{\partial^{2} V}{\partial X^{2}}[X]_{t} \\
d V\left(s_{t}, i_{t}, a_{t}, d_{t}, u_{t}\right) & =\mathcal{L} V d t+Q d B_{t}
\end{aligned}
$$

From system equation (2.2), let define $V_{1}\left(s_{t}, i_{t}\right)=e^{t}\left(s^{\theta}+i^{\theta}\right)$, then we have a drift part as:

$$
\begin{aligned}
& \mathcal{L} V_{1}=e^{t}\left(s^{\theta}+i^{\theta}\right)+e^{t}\left[\theta s^{\theta-1} d s+\frac{1}{2} \theta(\theta-1) s^{\theta-2}\left(\sigma_{1}^{2} i^{2} s^{2}\right)+\theta i^{\theta-1} d i+\right. \\
& \left.\quad \frac{1}{2} \theta(\theta-1) i^{\theta-2}\left(\sigma_{1}^{2} i^{2} s^{2}\right)\right] \\
& \quad=e^{t}\left[s^{\theta}+i^{\theta}+\theta s^{\theta-1}(\rho \Lambda-(\beta i+\Lambda-\alpha(a+d+u)) s)+\frac{1}{2} \theta(\theta-1) s^{\theta-2}\left(\theta_{1}^{2} i^{2} s^{2}\right)+\right. \\
& \\
& \left.\theta i^{\theta-1}\left((1-\rho) \Lambda+\left(\beta s-\theta_{1}-\Lambda+\alpha(a+d+u)\right) i\right)+\frac{1}{2} \theta(\theta-1) i^{\theta-2}\left(\theta_{1}^{2} i^{2} s^{2}\right)\right]
\end{aligned}
$$

Similarly, let $V_{2}\left(a_{t}, d_{t}, u_{t}\right)=e^{t}\left[a^{\theta}+d^{\theta}+u^{\theta}\right]$

$$
\begin{aligned}
& \mathcal{L} V_{2}=e^{t}\left(a^{\theta}+d^{\theta}+u^{\theta}\right)+e^{t}\left[\theta a^{\theta-1} d a+\frac{1}{2} \theta(\theta-1) a^{\theta-2}\left(\sigma_{2}^{2} a^{2}\right)+\theta d^{\theta-1} d \mathrm{~d}+\right. \\
& \left.\quad \frac{1}{2} \theta(\theta-1) d^{\theta-2}\left(\sigma_{2}^{2} \psi^{2} a^{2}\right)+\theta u^{\theta-1} d u+\frac{1}{2} \theta(\theta-1) u^{\theta-2}(1-\psi)^{2} \sigma_{2}^{2} a^{2}\right] \\
& \quad=e^{t}\left[\left(a^{\theta}+d^{\theta}+u^{\theta}\right)+\theta a^{\theta-1}\left(\theta_{1} i+\theta_{2} d+\theta_{3} u-(\alpha+\omega+\Lambda-\alpha(a+d+u)) a\right)+\right. \\
& \\
& \quad \frac{1}{2} \theta(\theta-1) a^{\theta-2}\left(\sigma_{2}^{2} a^{2}\right)+\theta d^{\theta-1}\left(\psi \omega a-\left(\alpha+\theta_{2}+\Lambda-\alpha(a+d+u)\right) d\right)+ \\
& \\
& \frac{1}{2} \theta(\theta-1) d^{\theta-2}\left(\sigma_{2}^{2} \psi^{2} a^{2}\right)+\theta u^{\theta-1}\left((1-\psi) \omega a-\left(\alpha+\theta_{3}+\Lambda-\alpha(a+d+u)\right) u\right)+ \\
& \\
& \left.\frac{1}{2} \theta(\theta-1) u^{\theta-2}(1-\psi)^{2} \sigma_{2}^{2} a^{2}\right]
\end{aligned}
$$

Thus, the drift part can be rewritten as

$$
\mathcal{L} V d t=\left(\mathcal{L} V_{1}+\mathcal{L} V_{2}\right) e^{t}
$$

The diffusion part fo the system (2.2) is given as:

$$
\begin{aligned}
& Q=-e^{t} \theta s^{\theta-1} \sigma_{1} i s d B_{1}(t)+e^{t} \theta i^{\theta-1} \sigma_{1} i s d B_{1}(t)-e^{t} \theta a^{\theta-1} \sigma_{1} \sigma_{2} a d B_{2}(t)+ \\
& e^{t} \theta d^{\theta-1} \sigma_{1} \sigma_{2} \psi a d B_{2}(t)+e^{t} \theta u^{\theta-1} \sigma_{1} \sigma_{2}(1-\psi) a d B_{2}(t) \\
& Q=e^{t} \theta\left[\sigma_{1} i s\left(s^{\theta-1}+i^{\theta-1}\right) d B_{1}(t)-\sigma_{1} \sigma_{2} a\left(a^{\theta-1}-\psi d^{\theta-1}-(1-\psi) u^{\theta-1}\right) d B_{2}(t)\right]
\end{aligned}
$$

Substituting (3.12) and (3.13) into (3.11) we have,

$$
\begin{aligned}
& d V=e^{t} \mathcal{L} V d t+e^{t} Q d B_{t} \\
& d V=C e^{t} d t+e^{t} \theta\left[\sigma_{1} i s\left(s^{\theta-1}+i^{\theta-1}\right) d B_{1}(t)-\sigma_{1} \sigma_{2} a\left(a^{\theta-1}-\right.\right. \\
& \left.\left.\quad \psi d^{\theta-1}-(1-\psi) u^{\theta-1}\right) d B_{2}(t)\right]
\end{aligned}
$$


Integrating on both sides $\left[0, t \wedge \tau_{k}\right]$, we get

$$
\begin{gathered}
\int_{0}^{t \wedge \tau_{k}} d V=\int_{0}^{t \wedge \tau_{k}} C e^{t} d s+\int_{0}^{t \wedge \tau_{k}} e^{t} \theta\left[\sigma_{1} i s\left(s^{\theta-1}+i^{\theta-1}\right) d B_{1}(s)-\right. \\
\left.\sigma_{1} \sigma_{2} a\left(a^{\theta-1}-\psi d^{\theta-1}-(1-\psi) u^{\theta-1}\right) d B_{2}(s)\right] \\
V\left(s_{t}, i_{t}, a_{t}, d_{t}, u_{t}\right)=V(s(0), i(0), a(0), d(0), u(0))+\int_{0}^{t \wedge \tau_{k}} C e^{t} d s+ \\
\int_{0}^{t \wedge \tau_{k}} e^{t} \theta\left[\sigma_{1} i s\left(s^{\theta-1}+i^{\theta-1}\right) d B_{1}(s)-\sigma_{1} \sigma_{2} a\left(a^{\theta-1}-\psi d^{\theta-1}-\right.\right. \\
\left.\left.(1-\psi) u^{\theta-1}\right) d B_{2}(s)\right]
\end{gathered}
$$

where $C$ is a suitable constant, then taking expectation (3.16) on both sides, we get

$$
\begin{gathered}
\mathbb{E}\left[V\left(s_{t \wedge \tau_{k}}, i_{t \wedge \tau_{k}}, a_{t \wedge \tau_{k}}, d_{t \wedge \tau_{k}}, u_{t \wedge \tau_{k}}\right)\right] \leq \mathbb{E}[V(s(0), i(0), a(0), d(0), u(0))]+ \\
C \mathbb{E} \int_{0}^{t \wedge \tau_{k}} e^{t} d s
\end{gathered}
$$

Upon letting $k \longrightarrow \infty$, we get

$$
\begin{aligned}
& \mathbb{E}\left[V\left(s_{t}, i_{t}, a_{t}, d_{t}, u_{t}\right)\right] \leq V(s(0), i(0), a(0), d(0), u(0))+C\left(e^{t}-1\right) \\
& \text { which implies that } \\
& \mathbb{E}\left[V\left(s_{t}, i_{t}, a_{t}, d_{t}, u_{t}\right)\right] \leq e^{-t} V(s(0), i(0), a(0), d(0), u(0))+C
\end{aligned}
$$

Note that,

$$
\begin{aligned}
& |X(t)|^{\theta}=\left(s^{2}+i^{2}+a^{2}+d^{2}+u^{2}\right)^{\frac{\theta}{2}} \\
& |X(t)| \leq 5^{\frac{\theta}{2}} \max \left\{s^{\theta}, i^{\theta}, a^{\theta}, d^{\theta}, u^{\theta}\right\} \\
& |X(t)| \leq 5^{\frac{\theta}{2}}\left\{s^{\theta}+i^{\theta}+a^{\theta}+d^{\theta}+u^{\theta}\right\}
\end{aligned}
$$

Then we get,

$$
\mathbb{E}|X(t)|^{\theta} \leq 5^{\frac{\theta}{2}}\left(e^{-t} V(s(0), i(0), a(0), d(0), u(0))+C\right)
$$

which means that,

$$
\limsup _{t \rightarrow \infty} \mathbb{E}|X(t)|^{\theta} \leq 5^{\frac{\theta}{2}} C
$$

and it follows that

$$
\begin{aligned}
& \limsup _{t \rightarrow \infty} \mathbb{E}|X(t)|^{\theta} \leq 5^{\frac{\theta}{2}} C \leq \mathbf{k}(\theta)<\infty \\
& \limsup _{t \rightarrow \infty} \mathbb{E}|X(t)|^{\theta} \leq \mathbf{k}(\theta)<\infty
\end{aligned}
$$

where $\mathbf{k}(\theta)=5^{\frac{\theta}{2}} C$.

Using the above lemma (3.3) we showed that the solution of system (2.2) is stochastically ultimately bounded.

Theorem 3.2. For any initial value $X(0) \in \Gamma$, the solution of the system (2.2) is stochastically ultimate bounded

Proof. $\exists$ a positive constant $\delta_{1}$ such that

$$
\limsup _{t \rightarrow \infty} \mathbb{E}|\sqrt{X(t)}|<\delta_{1}
$$

For any $\epsilon>0$, and upon setting $\delta=\frac{\delta_{1}^{2}}{\epsilon^{2}}$, then by using Chebyshev's inequality we get,

$$
\mathbb{P}\{|X(t)|>\delta\} \leq \frac{\mathbb{E}|\sqrt{X(t)}|}{\sqrt{\delta}}
$$


Thus, it follows that

$$
\limsup _{t \rightarrow \infty} \mathbb{P}\{|\sqrt{X(t)}|>\delta\} \leq \frac{\delta_{1}}{\sqrt{\delta}}=\epsilon
$$

\subsection{Stochastic Extinction of the Disease}

We will focus on the conditions which guarantee the extinction of disease $i(t)$. We are going to explore the conditions which lead to the extinction of diseases of the system (2.2) under a white noise stochastic disturbance. Consider the following theorem regarding the extinction of disease for stochastic model.

Theorem 3.3. For any initial value $(s(0), i(0), a(0), d(0), d(0)) \in \mathfrak{R}_{+}^{5}$, the system (2.2) has a unique positive solution $(s(t)+i(t)+a(t)+d(t)+u(t)) \in \mathfrak{R}_{+}^{5}$ for $t \geq 0$ a.s.

Then the solution of the system $(s(t)+i(t)+a(t)+d(t)+u(t))$ following properties: If $R_{0}=\frac{\beta \rho}{\left(\Lambda+\theta_{1}\right)}<1$ and

$$
\sigma=\frac{\beta}{\sqrt{2\left(\Lambda+\theta_{1}\right)}} \quad \text { or } \quad \sigma^{2}=\frac{\beta^{2}}{2\left(\Lambda+\theta_{1}\right)}
$$

then,

$$
\lim _{t \rightarrow \infty} \frac{i(t)}{t}=0 \quad \text { a.s }
$$

and

$$
\limsup _{t \rightarrow \infty} \frac{\ln i(t)}{t} \leq 0 \quad \text { a.s }
$$

also

$$
\lim _{t \rightarrow \infty} \frac{\int_{0}^{t} i(t) d B_{1}(r)}{t}=0 \quad a . s
$$

Theorem 3.4. Assume that $\sigma_{2}^{2}=\frac{\beta^{2}}{2\left(\Lambda+\theta_{1}\right)}$. Let $(s(t)+i(t)+a(t)+d(t)+u(t)) \in \mathfrak{R}_{+}$be the solution of system (2.2) with initial values $(s(0)+i(0)+a(0)+d(0)+u(0)) \in \mathfrak{R}_{+}^{5}$

If $R_{0}=\frac{\beta \rho}{\left(\Lambda+\theta_{1}+\frac{\sigma_{1}^{2}}{2}\right)}<1$ then,

$$
\limsup _{t \rightarrow \infty} \frac{\ln i(t)}{t} \leq\left(\Lambda+\theta_{1}+\frac{1}{2} \sigma_{1}^{2}\right)\left(R_{0}^{s}-1\right)<0 \quad \text { a.s }
$$

which means the disease dies out with probability one.

Proof. From the first equation of the system (2.2), we have

$$
\frac{s(t)-s(0)}{t}=[\rho \Lambda-(\beta\langle i\rangle+\Lambda-\alpha(\langle a\rangle+\langle d\rangle+\langle u\rangle))\langle s\rangle]-\frac{\sigma_{1}}{t} \int_{0}^{t} i(r) s(r) d B_{1}(r)
$$

which means that

$$
\begin{aligned}
\frac{s(t)-s(0)}{t} & =\rho \Lambda-\beta\langle s\rangle\langle i\rangle-\Lambda\langle s\rangle+\alpha\langle s\rangle(\langle a\rangle+\langle d\rangle+\langle u\rangle)-\mathcal{M} \\
\langle s\rangle & \left.=\rho-\beta \frac{[\langle s\rangle\langle i\rangle+\alpha\langle s\rangle(\langle a\rangle+\langle d\rangle+\langle u\rangle)-}{\Lambda}\right]-\mathcal{M}-\frac{s(t)-s(0)}{\Lambda t} \\
\text { Let } \quad \mathcal{M} & =\frac{\sigma_{1}}{\Lambda t} \int_{0}^{t} i(r) s(r) d B_{1}(r)
\end{aligned}
$$


From (3.25) and (3.27) it follows that,

$$
\begin{gathered}
\lim _{t \rightarrow \infty} \mathcal{M}=0 \quad \text { a.s } \\
\text { Hence } \quad \limsup _{t \rightarrow \infty} \frac{s(t)}{t} \leq \rho \quad \text { a.s }
\end{gathered}
$$

By using Itô formula, from (2.2), let consider infected it is $C^{2,1}\left(\mathfrak{R}^{5} \times(0, \infty): \mathfrak{R}_{+}\right)$

$$
\begin{aligned}
d i & =\left[(1-\rho) \Lambda+\left(\beta s-\theta_{1}-\Lambda+\alpha(a+d+u)\right) i\right] d t+\sigma_{1} i s d B_{1}(t) \\
\text { Let } X_{t} & =\ln i_{t}, \frac{\partial X_{t}}{\partial t}=0, \frac{\partial X_{t}}{\partial i}=\frac{1}{i}, \frac{\partial X_{t}^{2}}{\partial i^{2}}=-\frac{1}{i^{2}} \\
d \ln i(t) & =\frac{1}{i_{t}} d i_{t}-\frac{1}{2}\left(\frac{1}{i(t)^{2}}\right)[d i]_{t} \\
{[d i]_{t} } & =-\frac{1}{2} \sigma_{1}^{2} s(t)^{2} d t \\
d \ln i(t) & =\frac{\left[(1-\rho) \Lambda+\left(\beta s-\theta_{1}-\Lambda+\alpha(a+d+u)\right) i\right] d t+\sigma_{1} i s d B_{1}(t)}{i}-\frac{1}{2} \sigma_{1}^{2} s(t)^{2} d t \\
d \ln i(t) & =\left[\beta s-\theta_{1}-\Lambda+\alpha(a+d+u)-\frac{1}{2} \sigma_{1}^{2} s(t)^{2}\right] d t+\sigma_{1} s d B_{1}(t)
\end{aligned}
$$

Integrating both sides from $[0, t]$ gives,

$$
\begin{aligned}
& \int_{0}^{t} d \ln i(t)=\int_{0}^{t}\left[\beta s-\theta_{1}-\Lambda+\alpha(a+d+u)-\frac{1}{2} \sigma_{1}^{2} s(t)^{2}\right] d r+\sigma_{1} \int_{0}^{t} s d B_{1}(r) \\
& \ln i(t)=\ln i(0)+\left[\beta s-\left(\theta_{1}+\Lambda+\frac{\sigma_{1}^{2}}{2}\right)+\frac{\sigma_{1}^{2}}{2}-\frac{\sigma_{1}^{2} s^{2}}{2}+\alpha(a+d+u)-\frac{1}{2} \sigma_{1}^{2} s(t)^{2}\right] t+ \\
& \frac{1}{t} \sigma_{1} \int_{0}^{t} s d B_{1}(r)
\end{aligned}
$$

Let $\mathcal{M}=\sigma_{1} \int_{0}^{t} s d B_{1}(r)$. Then, $\mathcal{M}$ is a martingale Khasminskii (2011). This implies that quadrartic variation of stochastic integral becomes:

$$
\langle\mathcal{M}, \mathcal{M}\rangle_{t}=\sigma_{1} \int_{0}^{t} s d B_{1}(r)=\sigma_{1}^{2} \rho^{2} t
$$

by the Strong Law of Large Numbers for martingales, we have

$$
\limsup _{t \rightarrow \infty} \frac{\langle\mathcal{M}, \mathcal{M}\rangle_{t}}{t}<\infty \quad \text { a.s } \Longrightarrow \quad \lim _{t \rightarrow \infty} \frac{\mathcal{M}}{t}=0 \quad \text { a.s }
$$

Upon dividing both sides by $t$ we have,

$$
\begin{aligned}
\frac{\ln i(t)}{t} & =\frac{\ln i(0)}{t}+\left[\beta s-\left(\theta_{1}+\Lambda+\frac{\sigma_{1}^{2}}{2}\right)+\frac{\sigma_{1}^{2}}{2}-\frac{\sigma_{1}^{2} s^{2}}{2}+\alpha(a+d+u)-\frac{1}{2} \sigma_{1}^{2} s(t)^{2}\right]+\frac{\mathcal{M}}{t} \\
\frac{\ln i(t)}{t} & =\frac{\ln i(0)}{t}+\left(\Lambda+\theta_{1}+\frac{1}{2} \sigma_{1}^{2}\right)\left(\frac{\beta s}{\left(\Lambda+\theta_{1}+\frac{1}{2} \sigma_{1}^{2}\right)}-1\right)+\frac{\sigma_{1}^{2}}{2}-\frac{\sigma_{1}^{2} s^{2}}{2}+\alpha(a+d+u)- \\
& \frac{1}{2} \sigma_{1}^{2} s(t)^{2}+\frac{\mathcal{M}}{t} \\
\frac{\ln i(t)}{t} & \leq\left(\Lambda+\theta_{1}+\frac{1}{2} \sigma_{1}^{2}\right)\left(R_{0}^{s}-1\right)+\frac{\mathcal{M}}{t}
\end{aligned}
$$

Taking the limit superior on both sides leads to

$$
\limsup _{t \rightarrow \infty} \frac{\ln i(t)}{t} \leq\left(\Lambda+\theta_{1}+\frac{1}{2} \sigma_{1}^{2}\right)\left(R_{0}^{s}-1\right)<0 \quad \text { a.s }
$$

which indicates that $\lim _{t \rightarrow \infty} \frac{i(t)}{t}=0$ then disease will go to extinction exponentially with probability one, then $\left(R_{0}^{s}-1\right)<0$ implying that $R_{0}^{s}<1$. We conclude that the number of infected population will also tend to zero exponentially almost surely. 


\subsection{Stochastic Persistence of the Disease}

In this subsection we will show the condition when the disease prevails, we shall investigate the persistence of the disease.

Theorem 3.5. If $\bar{R}_{0}^{S}=\frac{\beta \rho}{\frac{\sigma_{2}^{2}}{2}\left(\Lambda+\theta_{1}+\frac{\sigma_{1}^{2}}{2}\right)}>1$ then for any initial value $(s(0), i(0), a(0), d(0), u(0)) \in \mathfrak{R}_{+}^{5}$, the disease $i(t)$ of the model system (2.2) has the property that

$$
\liminf _{t \rightarrow \infty}\langle i(t)\rangle \geq \frac{\Lambda \sigma_{2}^{2}}{\beta^{2} \rho}\left(\tilde{R}_{0}^{S}-1\right)\left(\theta_{1}+\Lambda+\frac{\sigma_{1}^{2}}{2}\right) \quad \text { a.s }
$$

That is to say, the disease will prevail if $\bar{R}_{0}^{S}>1$

Proof. We employ the stochastic Lyapunov method, let set $V_{1}=-a_{1} \log s-\log i-\log a-\log d-\log u$ as our Lyapunov function, where positive constant $a_{1}$ to be determined later.

From the generalized Itô formula we have,

$$
\begin{aligned}
d V_{1} & =\mathcal{L} V_{1} d t+a_{1} \sigma_{1} i \mathrm{~dB}_{1}(t)-a_{1} \sigma_{1} s \mathrm{~dB}_{1}(t)+\sigma_{2} \mathrm{~dB}_{2}(t)-\frac{\sigma_{2} \psi a}{d} \mathrm{~dB}_{2}(t) \\
& -\frac{\sigma_{2}(1-\psi) a}{u} \mathrm{~dB}_{2}(t)
\end{aligned}
$$

where

$$
\begin{aligned}
\mathcal{L} V_{1} & =\mathcal{L}\left(-a_{1} \log s-\log i-\log a-\log d-\log u\right) \\
\mathcal{L} V_{1} & =-\frac{a_{1} \rho \Lambda}{s}-a_{1}(\alpha(-(a+d+u))+\beta i+\Lambda)-\frac{\Lambda(1-\rho)}{i}+\beta s-\theta_{1}-\Lambda+\alpha(a+d+u) \\
& -\frac{\theta_{2} d+\theta_{1} i+\theta_{3} u}{a}+\alpha+\omega+\Lambda-\alpha(a+d+u)-\frac{a \psi \omega}{d}+\alpha+\theta_{2}+\Lambda-\alpha(a+d+u)- \\
& \frac{a(1-\psi) \omega}{u}+\alpha+\theta_{3}+\Lambda-\alpha(a+d+u)-\frac{\sigma_{2}^{2}\left(a^{2} \psi^{2}\right)}{2 d^{2}}-\frac{\sigma_{2}^{2}\left(a^{2}\left(1-\psi^{2}\right)\right)}{2 u^{2}}+\frac{1}{2} a_{1}^{2} i^{2} \sigma_{1}^{2}- \\
& \frac{1}{2} \sigma_{1}^{2} s^{2}-\frac{\sigma_{2}^{2}}{2}
\end{aligned}
$$

Upon simplifying we have,

$$
\mathcal{L} V_{1} \leq \alpha a_{1}(a+d+u)-a_{1} \beta i-a_{1} \Lambda-a_{1} \Lambda+3 \alpha-\theta_{1}+\theta_{2}+\theta_{3}+2 \Lambda+\beta s-\frac{\sigma_{2}^{2}}{2}+\omega
$$

Adding and substracting $\frac{\sigma_{1}^{2}}{2}$, and let $a_{1}=\frac{\beta \rho}{\frac{2 \Lambda \sigma_{2}^{2}}{2}}$

$$
\begin{aligned}
& \mathcal{L} V_{1} \leq \alpha a_{1}(a+d+u)-a_{1} \beta i-2 a_{1} \Lambda+3 \alpha+\beta \mathrm{s}-\theta_{1}+\theta_{2}+\theta_{3}+3 \Lambda-\Lambda+\frac{\sigma_{1}^{2}}{2}- \\
& \quad \frac{\sigma_{1}^{2}}{2}+\omega \\
& \mathcal{L} V_{1} \leq \alpha a_{1}(a+d+u)+3 \alpha-\frac{2(\beta \rho) \Lambda}{\frac{2 \Lambda \sigma_{2}^{2}}{2}}+\beta \rho-\theta_{1}+\theta_{2}+\theta_{3}-\frac{\beta \beta \rho i}{\frac{2 \Lambda \sigma_{2}^{2}}{2}}+3 \Lambda-\Lambda+\frac{\sigma_{1}^{2}}{2}- \\
& \quad \frac{\sigma_{1}^{2}}{2}+\omega \\
& \mathcal{L} V_{1} \leq 3 \alpha-\left(\theta_{1}+\Lambda+\frac{\sigma_{1}^{2}}{2}\right)\left(1-\frac{\beta \rho}{\frac{1}{2} \sigma_{2}^{2}\left(\theta_{1}+\Lambda+\frac{\sigma_{1}^{2}}{2}\right)}\right)+\beta \rho+\theta_{2}+\theta_{3}+\frac{i\left(\beta^{2} \rho\right)}{\Lambda \sigma_{2}^{2}}+ \\
& \quad 3 \Lambda+\frac{\sigma_{1}^{2}}{2}+\omega \\
& \mathcal{L} V_{1} \leq-\left(1-\tilde{R}_{0}^{S}\right)\left(\theta_{1}+\Lambda+\frac{\sigma_{1}^{2}}{2}\right)+3 \alpha+\beta \rho+\theta_{2}+\theta_{3}+\frac{i\left(\beta^{2} \rho\right)}{\Lambda \sigma_{2}^{2}}+3 \Lambda+\frac{\sigma_{1}^{2}}{2}+\omega
\end{aligned}
$$


From (3.37) we have

$$
\begin{aligned}
d V_{1} \leq\left[-\left(1-\tilde{R}_{0}^{S}\right)\left(\theta_{1}+\Lambda+\frac{\sigma_{1}^{2}}{2}\right)+3 \alpha+\beta \rho+\theta_{2}+\theta_{3}+\frac{i\left(\beta^{2} \rho\right)}{\Lambda \sigma_{2}^{2}}+3 \Lambda+\frac{\sigma_{1}^{2}}{2}+\omega\right] \mathrm{dt}+ \\
a_{1} \sigma_{1} i \mathrm{~dB}_{1}(t)-a_{1} \sigma_{1} s \mathrm{~dB}_{1}(t)+\sigma_{2} \mathrm{~dB}_{2}(t)-\frac{\sigma_{2} \psi a}{d} \mathrm{~dB}_{2}(t)-\frac{\sigma_{2}(1-\psi) a}{u} \mathrm{~dB}_{2}(t) \\
d V_{1} \leq\left[-\left(1-\tilde{R}_{0}^{S}\right)\left(\theta_{1}+\Lambda+\frac{\sigma_{1}^{2}}{2}\right)+3 \alpha+\beta \rho+\theta_{2}+\theta_{3}+\frac{i\left(\beta^{2} \rho\right)}{\Lambda \sigma_{2}^{2}}+3 \Lambda+\frac{\sigma_{1}^{2}}{2}+\omega\right] \mathrm{dt} \\
\quad+\mathcal{M}(t)
\end{aligned}
$$

integrating both sides and dividing by $t$, we obtain

$$
\begin{aligned}
& \frac{V_{1}(s(t), i(t), a(t), d(t), u(t))-V_{1}(s(0), i(0), a(0), d(0), u(0))}{t} \leq \\
& -\left(1-\tilde{R}_{0}^{S}\right)\left(\theta_{1}+\Lambda+\frac{\sigma_{1}^{2}}{2}\right)+3 \alpha+\beta \rho+\theta_{2}+\theta_{3}+\frac{\langle i\rangle \beta^{2} \rho}{\Lambda \sigma_{2}^{2}}+3 \Lambda+\frac{\sigma_{1}^{2}}{2}+\omega+\frac{\mathcal{M}(t)}{t}
\end{aligned}
$$

We compute $\langle i\rangle$ in terms of others

$$
\begin{aligned}
-\frac{\langle i\rangle \beta^{2} \rho}{\Lambda \sigma_{2}^{2}} \leq & -\frac{V_{1}(s(t), i(t), a(t), d(t), u(t))-V_{1}(s(0), i(0), a(0), d(0), u(0))}{t}- \\
& \left(1-\tilde{R}_{0}^{S}\right)\left(\theta_{1}+\Lambda+\frac{\sigma_{1}^{2}}{2}\right)+3 \alpha+\beta \rho+\theta_{2}+\theta_{3}+3 \Lambda+\frac{\sigma_{1}^{2}}{2}+\omega+\frac{\mathcal{M}(t)}{t} \\
\frac{\langle i\rangle \beta^{2} \rho}{\Lambda \sigma_{2}^{2}} \geq & \left(1-\tilde{R}_{0}^{S}\right)\left(\theta_{1}+\Lambda+\frac{\sigma_{1}^{2}}{2}\right)-\left[3 \alpha+\beta \rho+\theta_{2}+\theta_{3}+3 \Lambda+\frac{\sigma_{1}^{2}}{2}+\omega\right]
\end{aligned}
$$

by the strong law of large numbers for martingale from (3.26) and (3.27) it follows that

$$
\begin{aligned}
& \lim _{t \rightarrow \infty}\left[\frac{V_{1}(s(t), i(t), a(t), d(t), u(t))-V_{1}(s(0), i(0), a(0), d(0), u(0))}{t}\right]=0 \quad \text { a.s } \\
& \quad \text { where } \mathcal{M}(t)=\int_{0}^{t}\left[a_{1} \sigma_{1} i \mathrm{~dB}_{1}(t)-a_{1} \sigma_{1} s \mathrm{~dB}_{1}(t)+\sigma_{2} \mathrm{~dB}_{2}(t)-\frac{\sigma_{2} \psi a}{d} \mathrm{~dB}_{2}(t)-\right. \\
& \left.\quad \frac{\sigma_{2}(1-\psi) a}{u} \mathrm{~dB}_{2}(t)\right]
\end{aligned}
$$

Regarding the quadratic variations of the stochastic integral, this implies that the quadrartic variation is given as:

$$
\begin{aligned}
\langle\mathcal{M}, \mathcal{M}\rangle_{t} & =\int_{0}^{t}\left[a_{1} \sigma_{1} i \mathrm{~dB}_{1}(t)-a_{1} \sigma_{1} s \mathrm{~dB}_{1}(t)+\sigma_{2} \mathrm{~dB}_{2}(t)-\frac{\sigma_{2} \psi a}{d} \mathrm{~dB}_{2}(t)-\frac{\sigma_{2}(1-\psi) a}{u} \mathrm{~dB}_{2}(t)\right] \\
& =a_{1}^{2} \sigma_{1}^{2} i^{2}-a_{1}^{2} \sigma_{1}^{2} s^{2}+\sigma_{2}^{2}-\left(\frac{\sigma_{2} \psi a}{d}\right)^{2}-\left(\frac{\sigma_{2}(1-\psi) a}{u}\right)^{2}
\end{aligned}
$$

by strong law of large numbers for martingales

$$
\lim _{t \rightarrow \infty} \frac{\mathcal{M}(t)}{t}=0 \quad \text { a.s }
$$

Applying limit infimum throughout

$$
\begin{aligned}
& \liminf _{t \rightarrow \infty}\left[\frac{\langle i\rangle \beta^{2} \rho}{\Lambda \sigma_{2}^{2}}\right] \geq\left(1-\tilde{R}_{0}^{S}\right)\left(\theta_{1}+\Lambda+\frac{\sigma_{1}^{2}}{2}\right)-\left[3 \alpha+\beta \rho+\theta_{2}+\theta_{3}+3 \Lambda+\frac{\sigma_{1}^{2}}{2}+\omega\right] \\
& \liminf _{t \rightarrow \infty}\left[\frac{\langle i\rangle \beta^{2} \rho}{\Lambda \sigma_{2}^{2}}\right] \geq\left(\tilde{R}_{0}^{S}-1\right)\left(\theta_{1}+\Lambda+\frac{\sigma_{1}^{2}}{2}\right)+\left[3 \alpha+\beta \rho+\theta_{2}+\theta_{3}+3 \Lambda+\frac{\sigma_{1}^{2}}{2}+\omega\right]
\end{aligned}
$$

thus,

$$
\liminf _{t \rightarrow \infty}\langle i(t)\rangle \geq \frac{\Lambda \sigma_{2}^{2}}{\beta^{2} \rho}\left(\tilde{R}_{0}^{S}-1\right)\left(\theta_{1}+\Lambda+\frac{\sigma_{1}^{2}}{2}\right) \quad \text { a.s }
$$

that is to say, the disease will prevail if $\bar{R}_{0}^{s}>1$, which is the required assertion.

Remark 3.6. It is noted that $R_{0}^{s}<R_{0}^{d}$ means the dieases dies out. Similarly, if $\tilde{R}_{0}^{d}>1$ then $R_{0}^{d}>1$ means the disease persists.That is to say that, if for stochastic model the disease persists, then it also persists for a deterministic model. 


\section{Numerical Simulations}

To illustrate the various theoretical results presented above, the system (2.2) is simulated for various sets of parameters. In this section, we give some numerical simulations to show the effect of noise on the dynamics of the SIADU models by using the Milstein numerical Method as mention by Higham (2001).

\subsection{Milstein Numerical Scheme}

The stochastic model model of model (2.2) can be rewritten as the following discretization equations:

$$
\left\{\begin{array}{c}
s_{k+1}=s_{k}+\left[\rho \Lambda-\left(\beta i_{k}+\Lambda-\alpha\left(a_{k}+d_{k}+u_{k}\right)\right) s_{k}\right] \Delta t-\sigma_{1} i_{k} s_{k} \sqrt{\Delta t} \eta_{k}+0.5 \sigma_{1}^{2} i_{k} s_{k}\left(\eta_{k}^{2}-1\right) \Delta t \\
i_{k+1}=i_{k}+\left[(1-\rho) \Lambda+\left(\beta s_{k}-\theta_{1}-\Lambda+\alpha\left(a_{k}+d_{k}+u_{k}\right)\right) i_{k}\right] \Delta t+\sigma_{1} i_{k} s_{k} \sqrt{\Delta t} \zeta_{k}+ \\
0.5 \sigma_{1}^{2} i_{k} s_{k}\left(\zeta_{k}^{2}-1\right) \Delta t \\
a_{k+1}=a_{k}+\left[\theta_{1} i_{k}+\theta_{2} d_{k}+\theta_{3} u_{k}-\left(\alpha+\omega+\Lambda-\alpha\left(a_{k}+d_{k}+u_{k}\right)\right) a_{k}\right] \Delta t-\sigma_{2} a_{k} \sqrt{\Delta t} \mathrm{z}_{k}+ \\
0.5 \sigma_{2}^{2} A_{k}\left(\mathrm{z}_{k}^{2}-1\right) \Delta t \\
d_{k+1}=d_{k}+\left[\psi \omega a_{k}-\left(\alpha+\theta_{2}+\Lambda-\alpha\left(a_{k}+d_{k}+u_{k}\right)\right) d_{k}\right] \Delta t+\sigma_{2} \psi a_{k} \sqrt{\Delta t} \xi_{k}+ \\
0.5 \sigma_{2}^{2} \psi^{2} A_{k}\left(\xi_{k}^{2}-1\right) \Delta t \\
u_{k+1}=u_{k}+\left[(1-\psi) \omega a_{k}-\left(\alpha+\theta_{3}+\Lambda-\alpha\left(a_{k}+d_{k}+u_{k}\right)\right) u_{k}\right] \Delta t+\sigma_{2}(1-\psi) a_{k} \sqrt{\Delta t} \mathrm{e}_{k}+ \\
0.5 \sigma_{2}^{2}(1-\psi)^{2} A_{k}\left(\mathrm{e}_{k}^{2}-1\right) \Delta t
\end{array}\right.
$$

where time increment $\Delta t>0$, and $\eta_{k}, \zeta_{k}, \mathrm{z}_{k}, \xi_{k}$ and $\mathrm{e}_{k}$ are $N(0,1)$ independent Gaussian random variables. In order to verify the analytical conditions mentioned in theorem (3.4) we choose the parameters as follows: $\theta_{1}=0.5, \theta_{2}=0.2, \theta_{3}=$ $0.1, \sigma_{1}=0.9, \sigma_{2}=0.8, \Lambda=0.8, \omega=0.4, \psi=0.3, \alpha=0.33, \rho=0.8, \beta=0.4$

\subsection{Disease Extinction}

For the stochastic model, we have stochastic reproduction number as:

$$
\begin{aligned}
R_{0}^{s} & =\frac{\beta \rho}{\left(\theta_{1}+\Lambda+\frac{\sigma_{1}^{2}}{2}\right)} \\
R_{0}^{s} & =\frac{0.4 \times 0.8}{0.5+0.8+\frac{0.9^{2}}{2}} \\
R_{0}^{s} & =0.1877<1
\end{aligned}
$$

thus, according to theorem (3.4), we conclude that for a given initial values $(s(0)=0.9, i(0)=0.7, a(0)=0.5, d(0)=$ $0.15, u(0)=0.35) \in \mathfrak{R}_{+}^{5}$ then $\mathrm{I}(\mathrm{t})$ obeys equation (3.35) as:

$$
\begin{aligned}
& \limsup _{t \rightarrow \infty} \frac{\ln i(t)}{t} \leq\left(\Lambda+\theta_{1}+\frac{1}{2} \sigma_{1}^{2}\right)\left(R_{0}^{s}-1\right)<0 \quad \text { a.s } \\
& \limsup _{t \rightarrow \infty} \frac{\ln i(t)}{t} \leq\left(\frac{0.4 \times 0.8}{0.5+0.8+\frac{0.9^{2}}{2}}\right)(0.1877-1) \\
& \limsup _{t \rightarrow \infty} \frac{\ln i(t)}{t} \leq-0.1523<0 \quad \text { a.s }
\end{aligned}
$$

That is to say that, $\mathrm{I}(\mathrm{t})$ will tend to zero exponentially with probability one as observed in figure (1). Therefore the stochastic model (2.2) has disease extinction with choise of stochastic noise intensities $\sigma_{1}=0.9$ and $\sigma_{2}=0.8$ showing that white noise is helpful for disease control. 


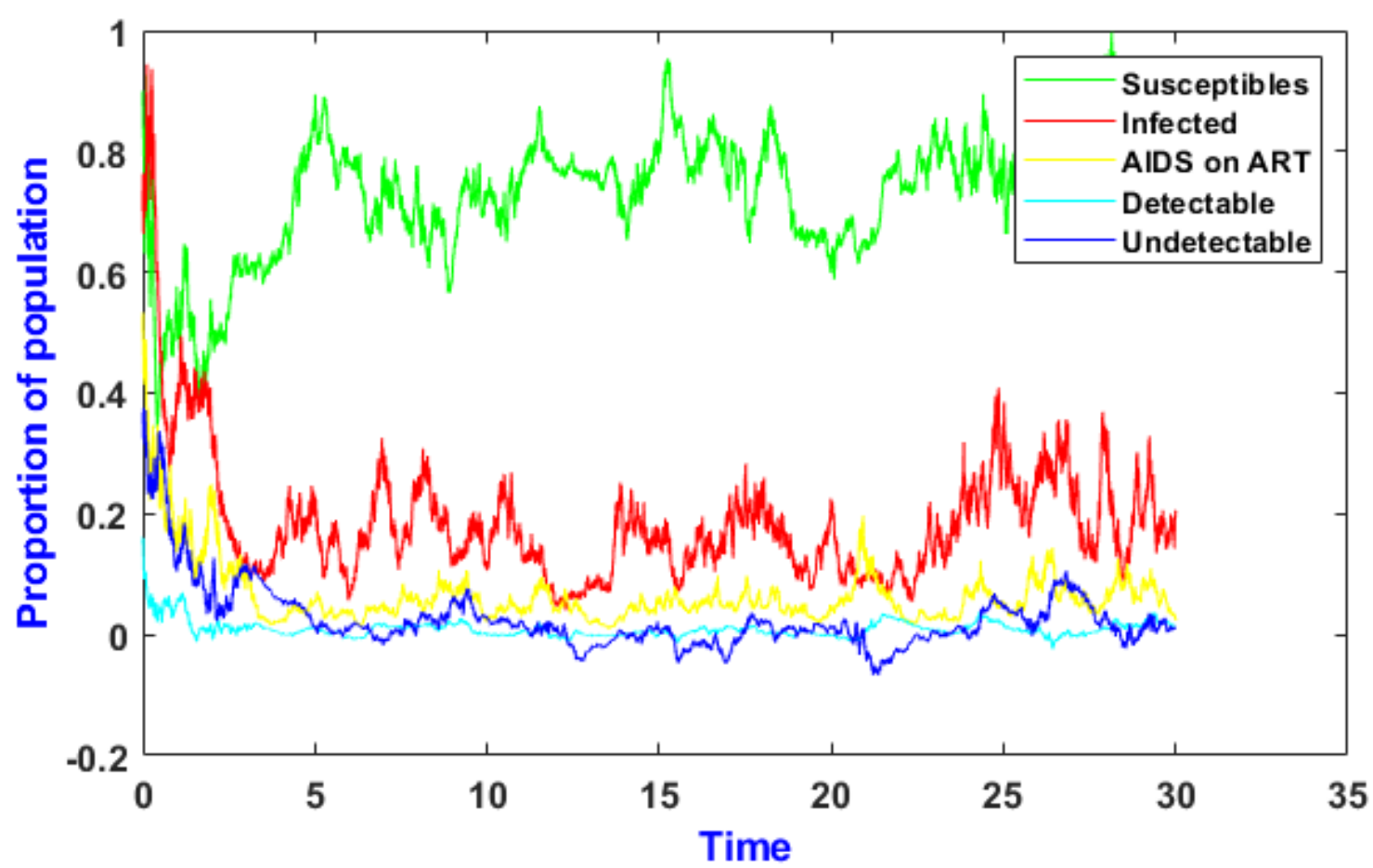

Figure 1. Stochastic trajectories for SIADU epidemic model, with $\sigma_{1}=0.9, \sigma_{2}=0.8$ with initial values $(s(0)=0.9, i(0)=0.7, a(0)=0.5, d(0)=0.15, u(0)=0.35)$ giving $R_{0}^{s}=0.1877<1$

\subsection{Disease Persistence}

To see the disease dynamics of model (2.2) we decrease the noise intensity say $\left(\sigma_{1}=0.2, \sigma_{2}=0.3\right), \theta_{1}=0.1$ and $\Lambda=0.4$ while keeping other parameters unaltered. Then from theorem (3.4) we have stochastic reproduction number as:

$$
\begin{aligned}
R_{0}^{s} & =\frac{\beta \rho}{\left(\Lambda+\theta_{1}+\frac{\sigma_{1}^{2}}{2}\right)} \\
R_{0}^{s} & =\frac{0.4 \times 0.7}{\left(0.4+0.1+\frac{0.2^{2}}{2}\right)} \\
R_{0}^{s} & =\frac{0.4 \times 0.7}{\left(0.4+0.1+\frac{0.2^{2}}{2}\right)}=1.2115>1
\end{aligned}
$$

Therefore, the condition of theorem (3.4) is not satisfied. In this case, numerical simulations suggest that $\mathrm{I}(\mathrm{t})$ is stochastically persistent and the disease will prevail as in figure (2). 


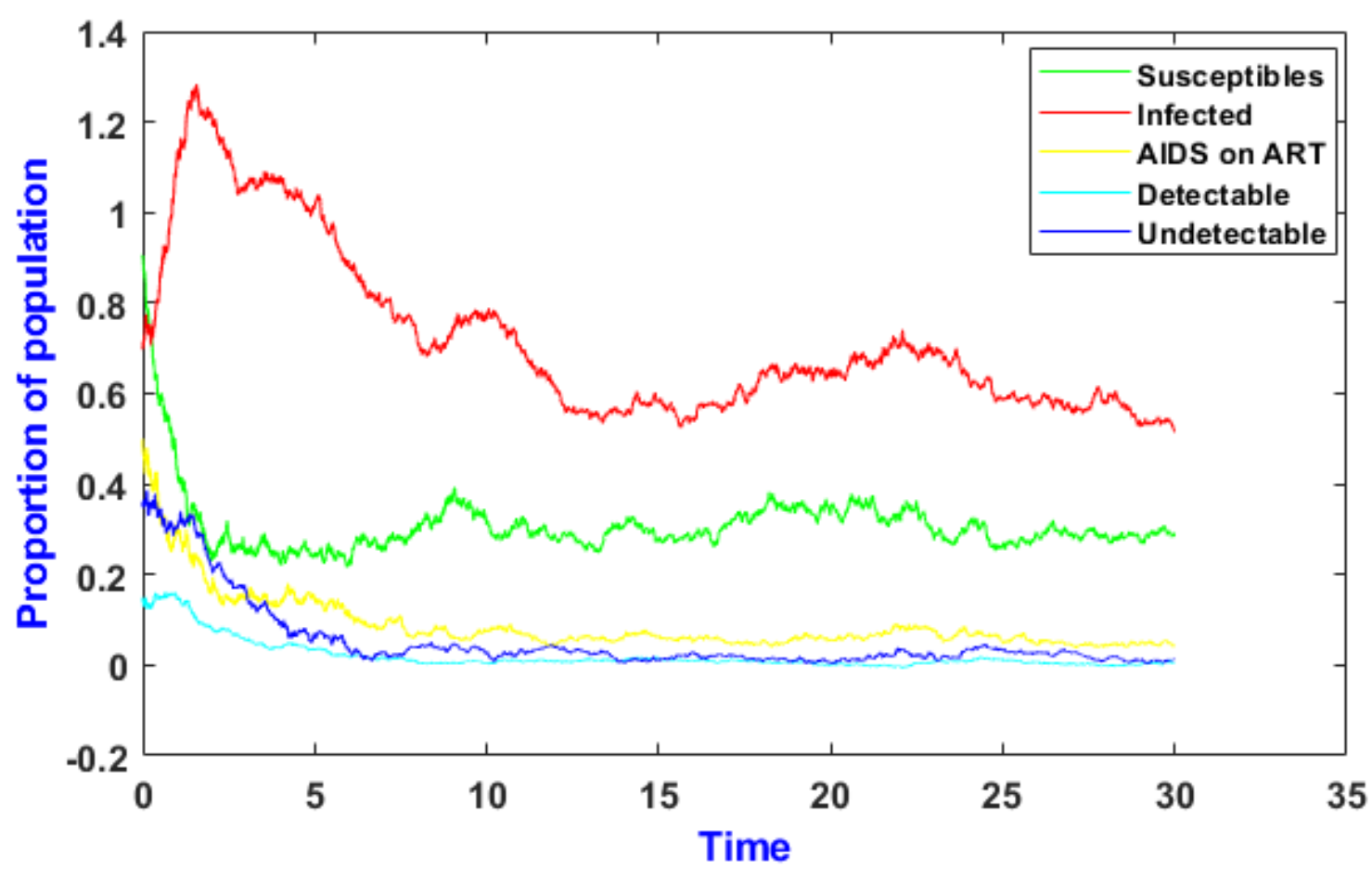

Figure 2. Stochastic trajectories for SIADU epidemic model with $\sigma_{1}=0.2, \Lambda=0.4, \theta_{1}=0.1$ with initial values $(s(0)=0.9, i(0)=0.7, a(0)=0.5, d(0)=0.15, u(0)=0.35)$ giving $R_{0}^{s}=1.2115>1$

\subsection{Stochastic vs Deterministic Model Solution}

\subsubsection{Comparing General Solution of the System}

Dynamic behaviaral comparisons between deterministic and the stochastic $S$ IADU model by investigating the basic reproduction number. For stochastic model system in (2.2) with parameter and the initial values as $(s(0)=0.9, i(0)=$ $0.7, a(0)=0.5, d(0)=0.15, u(0)=0.35) \in \mathfrak{R}_{+}^{5}$ we obained $R_{0}^{s}=0.1877<1$ while for the deterministic model (2.1) by using the same parameters, we obtained the reproduction number as $R_{0}=0.2462<1$. This shows that the stochastic model (2.2) supresses the disease outbreak.

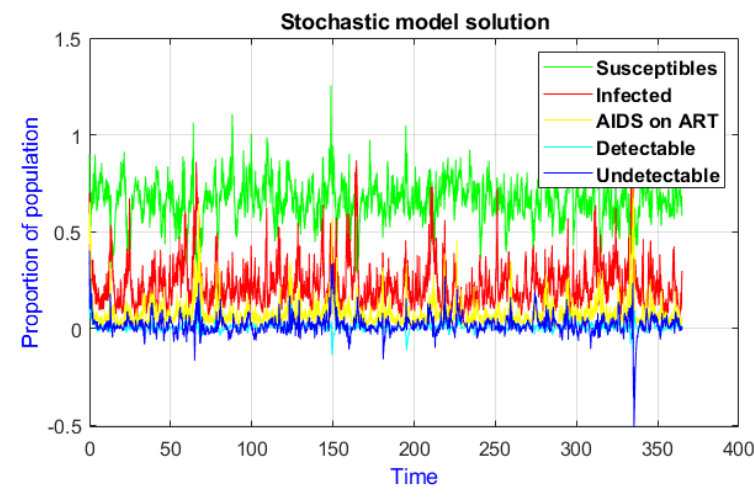

(a) Stochastic model with $R_{0}^{s}=0.1877<1$

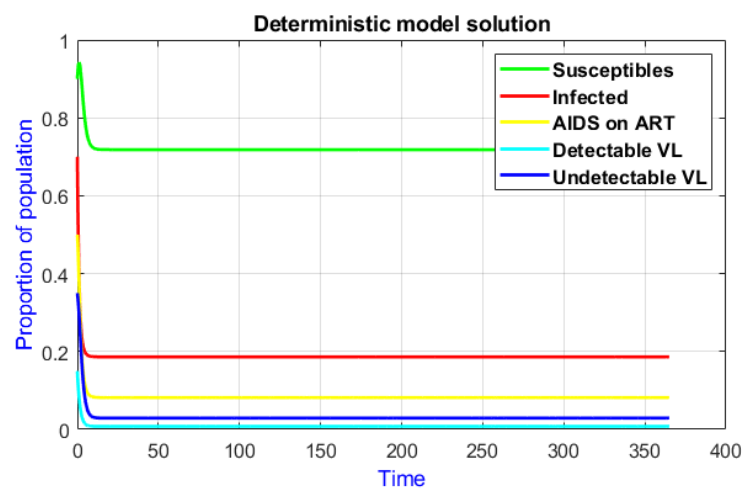

(b) Deterministic model with $R_{0}^{d}=0.2462<1$

Figure 3. The trajectories of the solution of system of stochastic model with $R_{0}^{s}=0.1877<1$ and Time evolutions of the deterministic SIADU model $R_{0}^{d}=0.2462<1$ 


\subsubsection{Comparing Individual Solution of the Models}
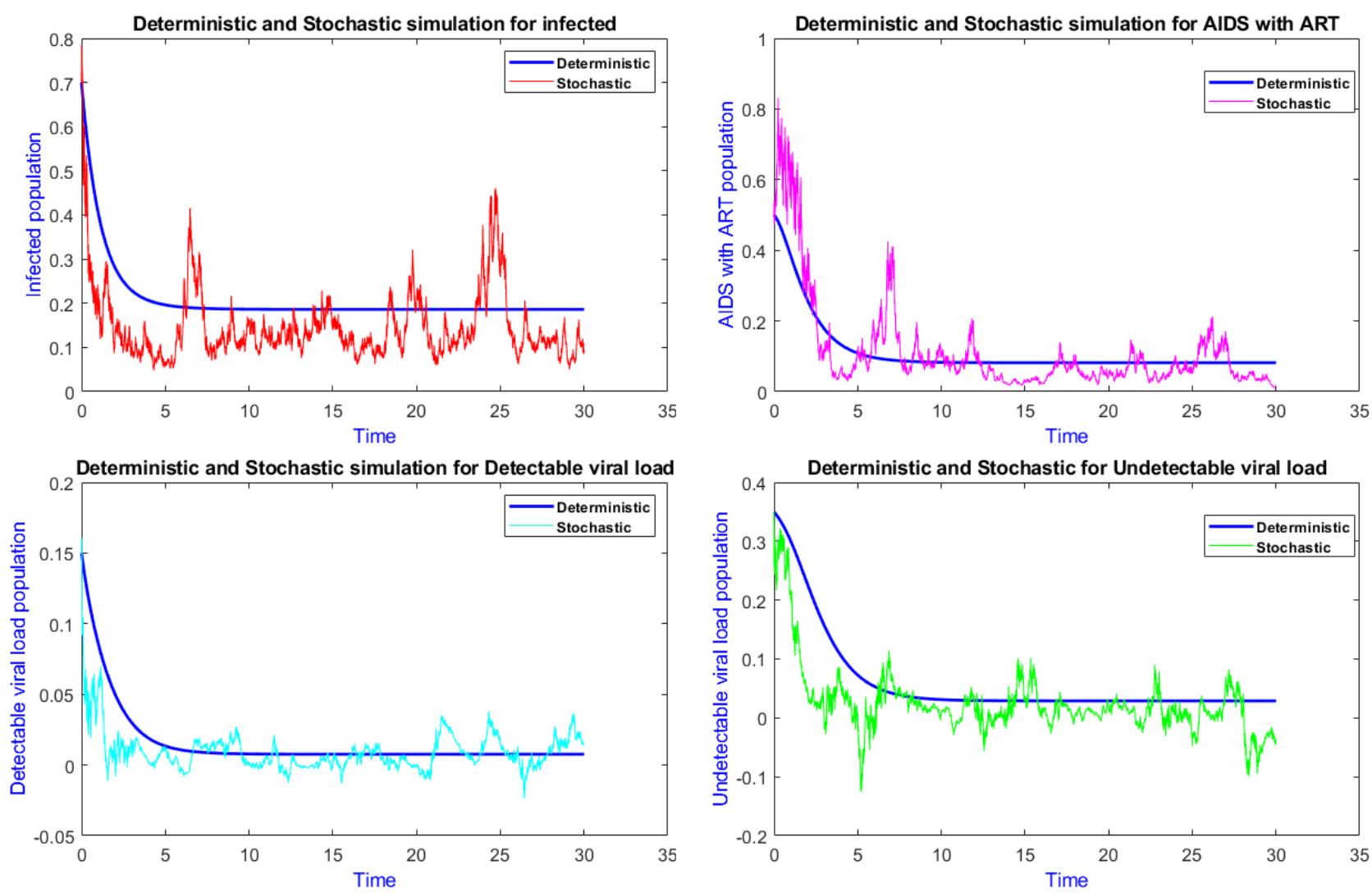

Figure 4. The trajectories of the individual solution for the stochastic model and Time evolutions of the deterministic SIADU model

\section{Concluding Remark}

In this paper, we discussed the properties of a stochastic model for HIV and AIDS by incorporating the viral load detectability, we derived from its deterministic counterpart. We investigated analytically the existence, uniqueness, and positivity of the stochastic model, similarly, we established sufficient conditions for stochastic disease extinction and persistence for the HIV and AIDS model. We used Milstein numerical method to obtain numerical results in order to illustrate the main analytical results, and by using stochastic basic reproduction number shown in theorem (3.4), we investigated the stochastic extinction as shown in figure (1) which exhibit diseases extinction with $R_{0}=0.4223<1$, showing that the disease dies out exponentially if the condition is met. Meanwhile using theorem (3.5) we showed that figure (2) exhibits diseases persistence with $R_{0}=1.2115>1$ that the disease will prevail in the population. Moreover, figure (3) and (4) clearly shows the stochastic trajectories and time evolution for the deterministic model. Additionally, with initial values $(s(0)=0.9, i(0)=0.7, a(0)=0.5, d(0)=0.15, u(0)=0.35)$ we investigated on the effects of noise strength intensity level, and the results revealed that the high noise intensity level results into disease suppression whilst on other hands low level of noise strength into the model does not suppress diseases.

Finally, the study concurs to the studies done by (Zhang and Zhou, 2019; Fan et al., 2017; Zhao and Yuan, 2016) showing that stochasticity in the model suppresses the outbreak of diseases. Therefore, as the reproduction number affected by random perturbation is smaller as compared to the reproduction number for the deterministic case, then the stochasticity present in the model (2.2) suppresses disease outbreak, hence useful for disease control and eradication.

\section{Acknowledgements}

I would like to express my heartfelt appreciation to Pan African University, Institute for Basic Sciences, Technology and Innovation(Kenya) for financial support and also I am grateful to the anonymous reviewers. 


\section{References}

Cai, Y., Kang, Y., Banerjee, M., \& Wang, W. (2016). A stochastic epidemic model incorporating media coverage. Communications in mathematical sciences, 14(4), 893-910. https://dx.doi.org/10.4310/CMS.2016.v14.n4.a1

Fan, K., Zhang, Y., Gao, S., \& Wei, X. (2017). A class of stochastic delayed sir epidemic models with generalized nonlinear incidence rate and temporary immunity. Physica A: Statistical Mechanics and its Applications, 481, 198208. https://doi.org/10.1016/j.physa.2017.04.055

Higham, D. J. (2001). An algorithmic introduction to numerical simulation of stochastic differential equations. SIAM review, 43(3), 525-546. https://doi.org/10.1137/S0036144500378302

Ji, C., \& Jiang, D. (2017). The extinction and persistence of a stochastic sir model. Advances in Difference Equations, (1), 1-8. https://doi.org/10.1186/s13662-016-1068-z

Khasminskii, R. (2011). Stochastic stability of differential equations. Springer Science \& Business Media. https://doi.org/10.1007/978-3-642-23280-0

Mao, X., Marion, G., \& Renshaw, E. (2002). Environmental brownian noise suppresses explosions in population dynamics. Stochastic Processes and their Applications, 97(1), 95-110. https://doi.org/10.1016/S0304-4149(01)00126-0

Mbogo, W. R., Livingstone, S. L., \& Odhiambo, J. W. (2013). Mathematical model for hiv and cd4+ cells dynamics in vivo. International Journal of Pure and Applied Mathematics, 6(2), 83-103. https://dx.doi.org/10.2139/ssrn.2225633

Miao, A., Wang, X., Zhang, T., Wang, W., \& Pradeep, B. S. A. (2017). Dynamical analysis of a stochastic sis epidemic model with nonlinear incidence rate and double epidemic hypothesis. Advances in Difference Equations, 2017(1), 226. https://doi.org/10.1186/s13662-017-1289-9

Nsengiyumva, A. C. et al. (2013). Numerical simulation of stochastic di erential equations. http://urn.fi/URN:NBN:fife201311217382

N'zi, M., \& Kanga, G. (2016). Global analysis of a deterministic and stochastic nonlinear sirs epidemic model with saturated incidence rate. Random Operators and Stochastic Equations, 24(1), 65-77. https://doi.org/10.1515/rose2016-0005

Song, Y., Miao, A., Zhang, T., Wang, X., \& Liu, J. (2018). Extinction and persistence of a stochastic sirs epidemic model with saturated incidence rate and transfer from infectious to susceptible. Advances in Difference Equations, (1), 1-11. https://doi.org/10.1186/s13662-018-1759-8

Tengaa, P. E., Mwalili, S., \& Orwa, G. O. (2020). Deterministic modeling for hiv and aids epidemics with viral load detectability. J. Math. Comput. Sci., 10(4), 728-757. https://doi.org/10.28919/jmcs/4474

Van Herwaarden, O. A., \& Grasman, J. (1995). Stochastic epidemics: major outbreaks and the duration of the endemic period. Journal of mathematical biology, 33(6), 581-601. https://doi.org/10.1007/BF00298644

Witbooi, P. J. (2013). Stability of an seir epidemic model with independent stochastic perturbations. Physica A: Statistical Mechanics and its Applications, 392(20), 4928-4936. https://doi.org/10.1016/j.physa.2013.06.025

Zhang, Q., \& Zhou, K. (2019). Extinction and persistence of a stochastic sirs model with nonlinear incidence rate and transfer from infectious to susceptible. In Journal of Physics: Conference Series, 1324, 012-016. IOP Publishing. https://doi.org/10.1088/1742-6596/1324/1/012016

Zhang, X.-B., Huo, H.-F., Xiang, H., \& Meng, X.-Y. (2014). Dynamics of the deterministic and stochastic siqs epidemic model with non-linear incidence. Applied Mathematics and Computation, 243, 546-558. https://doi.org/10.1016/j.amc.2014.05.136

Zhao, D., \& Yuan, S. (2016). Persistence and stability of the disease-free equilibrium in a stochastic epidemic model with imperfect vaccine. Advances in Difference Equations, 2016(1), 280. https://doi.org/10.1186/s13662-016-1010-4

\section{Copyrights}

Copyright for this article is retained by the author(s), with first publication rights granted to the journal.

This is an open-access article distributed under the terms and conditions of the Creative Commons Attribution license (http://creativecommons.org/licenses/by/4.0/). 